\title{
AUMENTO DA PRODUTIVIDADE DE SOJA COM A APLICAÇÃO DE BIOESTIMULANTES $\left({ }^{1}\right)$
}

\author{
DANILA COMELIS BERTOLIN $\left({ }^{*}\right)$; MARCO EUSTÁQUIO DE SÁ $\left({ }^{3}\right)$; ORIVALDO ARF $\left({ }^{3}\right)$; \\ ENES FURLANI JUNIOR $\left({ }^{3}\right)$; ADRIANA DE SOUZA COLOMBO $\left({ }^{2}\right)$; \\ FRANCIELLE LOUISE BUENO MELO DE CARVALHO $\left({ }^{2}\right)$
}

\begin{abstract}
RESUMO
A utilização de bioestimulantes proporciona incrementos no desenvolvimento vegetal embora poucos estudos tenham abordado aspectos fisiológicos da soja relacionados à aplicação destes produtos. Um experimento com a cultura da soja foi instalado com o objetivo de avaliar o uso de um bioestimulante composto por citocinina, ácido indolbutírico e ácido giberélico via sementes ou via foliar em diferentes estádios fenológicos de duas cultivares, sendo uma cultivar convencional (Conquista) e outra geneticamente modificada (Valiosa RR). Os tratamentos foram constituídos de cultivares, aplicações do produto via sementes na dose de $6 \mathrm{~mL}$ do produto comercial por quilo de sementes, via foliar na dose de $0,25 \mathrm{~L}$ do produto comercial por hectare, nos estádios $V_{5}, R_{1}$ e $R_{5}$, e a combinação desses fatores no total de 30 tratamentos para avaliar a altura de plantas, altura de inserção da primeira vagem, número de ramos por planta, de vagens por planta e de grãos por vagem. O delineamento experimental foi o de blocos ao acaso com quatro repetições. A cultivar convencional proporcionou maior produção de grãos do que a cultivar transgênica. A utilização do bioestimulante incrementou o número de vagens por planta e a produtividade de grãos, e os resultados para aplicação via sementes e via foliar não diferiram entre si. Na produtividade de grãos, o tratamento com bioestimulante proporcionou aumento de $37 \%$ em relação à testemunha. $\mathrm{O}$ bioestimulante aumenta o número de vagens por planta e produtividade de grãos tanto em aplicação via sementes quanto via foliar, confirmando a hipótese deste estudo. Todavia, a maior produtividade não está relacionada ao maior crescimento da parte aérea, considerando-se a altura das plantas, ramos por planta, altura de inserção da primeira vagem. Em relação ao aumento da produtividade, o bioestimulante é mais efetivo quando aplicado na fase reprodutiva.
\end{abstract}

Palavras-chave: fitorreguladores, fenologia, transgênica, Glycine max (L.) Merrill.

$\left({ }^{1}\right)$ Recebido para publicação em 5 de março de 2008 e aceito em 22 de dezembro de 2009. Parte da dissertação de mestrado de Danila Comelis Bertolin apresentada à Faculdade de Engenharia de Ilha Solteira, Universidade Estadual Paulista, para obtenção do título de mestre em Sistemas de Produção.

$\left({ }^{2}\right)$ Programa de Pós-graduação em Produção Vegetal da UNESP, campus de Ilha Solteira. Departamento de Fitotecnia, Tecnologia de Alimentos e Sócio-Economia, Faculdade de Engenharia, Universidade Estadual Paulista (Unesp), Caixa Postal 31, 15385-000 Ilha Solteira (SP), Brasil. E-mail: dcbertolin@aluno.feis.unesp.br $\left(^{*}\right)$ Autora correspondente. Bolsista CAPES.

$\left({ }^{3}\right)$ Departamento de Fitotecnia, Tecnologia de Alimentos e Sócio-Economia, Faculdade de Engenharia, Universidade Estadual Paulista (UNESP), Ilha Solteira, Ilha Solteira (SP), Brasil. 


\section{ABSTRACT \\ INCREASE OF THE PRODUCTIVITY OF THE SOYBEAN CROP WITH THE APPLICATION OF BIOSTIMULANTS}

The use of biostimulants increases plant development although few studies relate this practice to the physiological aspects of the plant. An experiment was carried out to evaluate the use of a biostimulant (cytokinin, indolebutyric acid, gibberellic acid), applied on soybean seeds and leaves at different phenological growth stages for a conventional variety (Conquista) and a genetically modified (Valiosa RR). The treatments were constituted by cultivars, applications of $6 \mathrm{~mL}$ of the commercial product per kilogram of seeds, and of $025 \mathrm{~L} \mathrm{ha}^{-1}$ on leaves at the stages $\mathrm{V}_{5}, \mathrm{R}_{1}$ and $\mathrm{R}_{5}$, and combination of these factors, comprising a total of 30 treatments. The experimental design was a randomized block with 4 replications. The height of plants, insertion of the first pod, number of branches per plant, number of pods per plant, and number of grains per pod were evaluated. The conventional variety was more productive than the genetically modified variety. The use of the biostimulant increased the number of pods per plant and seed yield although the application via seeds and on leaves was similar. The biostimulant increased grain yield by $37 \%$ compared to the control. Plant growth provides increase in the number of pods per plant and grain yield in both applications the leaves and seeds, confirming the hypothesis of this study. However, higher productivity is not related to increased shoot growth, considering the plant height, branches per plant, height of first pod. Regarding to the increase in $g$ the productivity plant growth, biostimulant is more, effective when applied during the reproductive growth stage.

Key words: plant regulators, phenology, transgenic plant, Glycine max (L.) Merrill.

\section{INTRODUÇÃO}

Alterações na concentração hormonal de tecidos podem mediar toda uma gama de processos de desenvolvimento das plantas, muitos dos quais envolvem interações com os fatores ambientais (Crozier et al. 2000). Na maioria das plantas, o crescimento da gema apical inibe o crescimento das gemas axilares, fenômeno denominado de dominância apical. A auxina, hormônio sintetizado nos meristemas apicais é responsável por este fenômeno, também pelo crescimento das plantas, influenciando diretamente nos mecanismos de expansão celular. As citocininas são ligadas à senescência foliar, mobilização de nutrientes, também à dominância apical, formação e atividade dos meristemas apicais e desenvolvimento floral. A função das giberelinas está associada à promoção do crescimento caulinar. Plantas submetidas a aplicação de giberelinas podem ser induzidas a obter maior crescimento na sua estatura (TAIZ e ZEIGER, 2004).

SANTos et al. (2005) analisaram doses de produto bioestimulante composto por citocinina, ácido indol butírico e ácido giberélico em aplicação via sementes em algodoeiro e observaram incremento na área foliar, altura e crescimento inicial de plantas. Segundo os autores, o bioestimulante aplicado via sementes é capaz de originar plântulas mais vigorosas, com maior comprimento, matéria seca e porcentagem de emergência em areia e terra vegetal proporcional ao aumento de doses do produto. Também CARVALHO et al. (1994), estudando a aplicação de fitorreguladores em algodoeiro concluíram que estes proporcionam aumento de peso do capulho e dos grãos.

Alguns pontos negativos são atribuídos ao uso de cultivares de soja resistentes ao glifosato. Para HARPER (1997) algumas cultivares têm baixo potencial genético para a produção, e em alguns casos, a baixa produtividade está relacionada com injúrias do herbicida na soja. De acordo com CASTRO (1981), poucos trabalhos abordam aspectos fisiológicos da planta de soja relacionados à aplicação de reguladores vegetais que são um manejo promissor para essa cultura. Informações sobre os efeitos destes produtos na soja poderiam fornecer elementos fundamentais para estudos posteriores sobre a utilização agronômica dos reguladores vegetais. Para LARCHER (2006), a ação de hormônios vegetais depende do estádio de desenvolvimento e da atividade da planta, de estímulos externos, da parte da planta que está recebendo o estímulo e do tempo deste impacto.

No Brasil, algumas culturas já atingiram altos níveis tecnológicos alcançando alta produtividade e já não estão condicionadas por limitações de ordem nutricional ou hídrica, o que tem levado ao emprego de biorreguladores, que podem ser compensadores além de econômicos (CASTRO, 2006).

Os hormônios vegetais são moléculas presentes em quantidades vestigiais, e mudanças na concentração hormonal e na sensibilidade dos tecidos podem mediar uma ampla gama de processos de desenvolvimento nas plantas, muitos 
dos quais envolvem interações biossintéticas, catabólicas que, juntas, controlam a homeostase dos hormônios vegetais (CROzIER, 2000). Os efeitos isolados dos hormônios vegetais foram bastante estudados e já conhecidos, sendo apresentados efeitos positivos e negativos de acordo com as quantidades aplicadas, períodos de aplicação, região de aplicação e culturas. No entanto, o efeito de alguns hormônios em conjunto é desconhecido, e visto das propriedades promissoras destas moléculas em culturas que já atingiram alto nível tecnológico são necessários maiores estudos.

A hipótese deste estudo é de que o bioestimulante promoverá aumento da produtividade da soja devido ao maior crescimento da planta. Adicionalmente, a ação dos hormônios vegetais é variável conforme o estádio de desenvolvimento, sendo mais efetiva logo na emergência das plântulas e no desenvolvimento inicial.

Tendo em vista a obtenção de aumento de produtividade, este estudo objetivou avaliar a produção de grãos de soja em função da aplicação de um bioestimulante com a seguinte composição: 0,009\% de cinetina, 0,005\% de ácido giberélico e 0,005\% de ácido indolbutírico, aplicado via sementes e via foliar em três estádios fenológicos da cultura, $V_{5}, R_{1}$ e $R_{5}$, em duas cultivares, sendo uma cultivar convencional (Conquista) e a outra geneticamente modificada (Valiosa RR).

\section{MATERIAL E MÉTODOS}

O experimento foi instalado em 23 de novembro de 2006, na Fazenda Experimental de Ensino, Pesquisa e Extensão da Faculdade de Engenharia de Ilha Solteira, localizada no município de Selvíria (MS). O solo foi classificado como Latossolo Vermelho Distrófico, argiloso (EMBRAPA, 1999). As características químicas do solo da área experimental na camada de $0-20 \mathrm{~cm}$ de profundidade foram as seguintes: M.O. $24 \mathrm{~g} \mathrm{dm}^{-3} ; \mathrm{pH} \mathrm{CaCl}_{2}$ 5,3; P resina $18 \mathrm{mg} \mathrm{dm}^{-3} ; \mathrm{V} \% 64 \mathrm{e} \mathrm{K}, \mathrm{Ca}, \mathrm{Mg}, \mathrm{H}+\mathrm{Al}$, e $\mathrm{Al}$ respectivamente 2,$2 ; 33 ; 14 ; 28$ e $0 \mathrm{mmolc} \mathrm{dm}^{-3}$, valores considerados adequados para a cultura (MASCARENHAS e TANAKA, 1997). A área foi cultivada com milho nos dois anos agrícolas anteriores e antes da semeadura da cultura de soja foi aplicado $250 \mathrm{~kg} \mathrm{ha}^{-1} \mathrm{da}$ formulação NPK (08-28-16).

O fornecimento de água, quando necessário, foi efetuado por aspersão e os dados médios diários de temperatura, umidade relativa do ar e de precipitação pluvial ocorridos durante o ciclo da cultura estão na figura 1 . A precipitação pluvial ocorrida durante o ciclo foi de $926 \mathrm{~mm}$ e a temperatura e umidade relativa média do ar, de $26,4^{\circ} \mathrm{C}$ e $80,7 \%$, respectivamente, estão de acordo com as necessidades da cultura para um rendimento satisfatório (EMBRAPA, 1996).

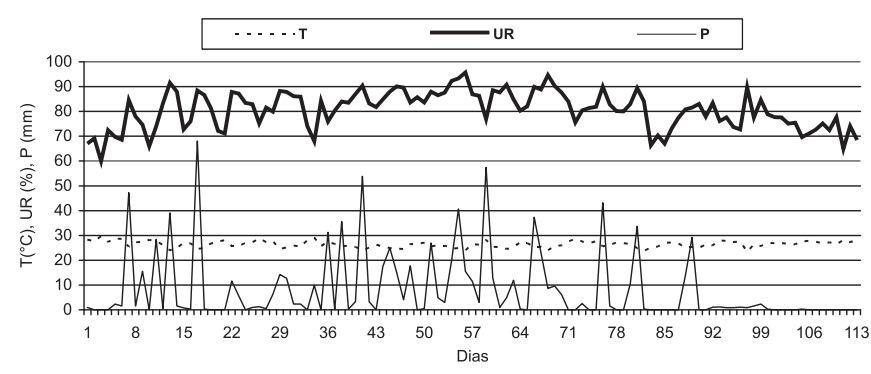

Figura 1. Médias diárias de temperatura do ar (T), umidade relativa do ar (UR) e precipitação total diárias (P) durante o ciclo da soja em Selvíria (MS), 2006/2007.

Foram utilizadas sementes de duas cultivares, uma convencional de ciclo médio, Conquista, e outra geneticamente modificada, Valiosa RR.

O espaçamento foi de $0,50 \mathrm{~m}$ entre linhas e a densidade calculada para 300.000 plantas por hectare. As sementes foram submetidas à inoculação com Bradyrhizobium, específico para sementes de soja, na dose de $250 \mathrm{~g}$ por $60 \mathrm{~kg}^{-1}$ de sementes. A semeadura foi realizada manualmente e os tratamentos via sementes foram aplicados pouco antes da semeadura. As parcelas tinham seis linhas de $5 \mathrm{~m}$, e a área útil de quatro linhas centrais a $0,5 \mathrm{~m}$ de cada extremidade. O experimento foi mantido livre de pragas através de controle químico e de plantas daninhas através de capina manual.

O bioestimulante é composto por três hormônios vegetais: $0,009 \%$ de cinetina, 0,005 $\%$ de ácido giberélico e $0,005 \%$ de ácido indolbutírico, e disponível com o nome comercial de Stimulate ${ }^{\circledR}$.

As aplicações do bioestimulante foram realizadas via sementes, pouco antes da semeadura, e depois por via foliar em três estádios de desenvolvimento, sendo um estádio vegetativo $\left(\mathrm{V}_{5}\right)$, e dois reprodutivos $\left(R_{1}\right.$ e $\left.R_{5}\right)$. As combinações destes fatores, constituíram 15 tratamentos por cultivar (Tabela 1), perfazendo total de 30 tratamentos.

O delineamento utilizado foi o de blocos ao acaso com quatro repetições. A dose utilizada na aplicação via sementes foi de $6 \mathrm{~mL}$ do produto comercial por quilo de sementes, a dose aplicada via foliar foi a de $0,25 \mathrm{~L}$ do produto comercial por hectare $(2,5 \mathrm{~mL}$ do produto comercial por litro de água). 
Tabela 1. Tratamentos realizados em cada cultivar. Selvíria (MS), 2006/2007

\begin{tabular}{|c|c|c|c|c|}
\hline \multirow{3}{*}{ Tratamentos } & \multicolumn{4}{|c|}{ Modo de aplicação do Bioestimulante } \\
\hline & \multirow{2}{*}{$\begin{array}{c}\text { Via sementes } \\
\text { TS }\end{array}$} & \multicolumn{3}{|c|}{ Via foliar } \\
\hline & & Fase $\mathrm{V}_{5}$ & Fase $R_{1}$ & Fase $R_{5}$ \\
\hline 01. Testemunha & - & - & - & - \\
\hline 02. TS & $6 \mathrm{~mL} \mathrm{pc} \mathrm{kg}^{-1}$ & - & - & - \\
\hline 03. $\mathrm{V}_{5}$ & - & $0,25 \mathrm{~L} \mathrm{pc} \mathrm{ha}^{-1}$ & - & - \\
\hline 04. $R_{1}$ & - & - & $0,25 \mathrm{~L} \mathrm{pc} \mathrm{ha}^{-1}$ & - \\
\hline 05. $R_{5}$ & - & - & - & $0,25 \mathrm{~L} \mathrm{pc} \mathrm{ha}^{-1}$ \\
\hline 06. $\mathrm{TS}+\mathrm{V}_{5}$ & $6 \mathrm{~mL} \mathrm{pc} \mathrm{kg}{ }^{-1}$ & $0,25 \mathrm{~L} \mathrm{pc} \mathrm{ha}^{-1}$ & - & - \\
\hline 07. $\mathrm{TS}+\mathrm{R}_{1}$ & $6 \mathrm{~mL} \mathrm{pc} \mathrm{kg}{ }^{-1}$ & - & $0,25 \mathrm{~L} \mathrm{pc} \mathrm{ha}^{-1}$ & - \\
\hline 08. $\mathrm{TS}+\mathrm{R}_{5}$ & $6 \mathrm{~mL} \mathrm{pc} \mathrm{kg}^{-1}$ & - & - & $0,25 \mathrm{~L} \mathrm{pc} \mathrm{ha}^{-1}$ \\
\hline 09. $V_{5}+R_{1}$ & - & $0,25 \mathrm{~L} \mathrm{pc} \mathrm{ha}^{-1}$ & $0,25 \mathrm{~L} \mathrm{pc} \mathrm{ha}^{-1}$ & - \\
\hline 10. $V_{5}+R_{5}$ & - & $0,25 \mathrm{~L} \mathrm{pc} \mathrm{ha}^{-1}$ & - & $0,25 \mathrm{~L} \mathrm{pc} \mathrm{ha}^{-1}$ \\
\hline 11. $R_{1}+R_{5}$ & - & - & $0,25 \mathrm{~L} \mathrm{pc} \mathrm{ha}^{-1}$ & $0,25 \mathrm{~L} \mathrm{pc} \mathrm{ha}^{-1}$ \\
\hline 12. $\mathrm{TS}+\mathrm{V}_{5}+\mathrm{R}_{1}$ & $6 \mathrm{~mL} \mathrm{pc} \mathrm{kg-1}$ & $0,25 \mathrm{~L} \mathrm{pc} \mathrm{ha}^{-1}$ & $0,25 \mathrm{~L} \mathrm{pc} \mathrm{ha}^{-1}$ & - \\
\hline 13. $\mathrm{TS}+\mathrm{V}_{5}+\mathrm{R}_{5}$ & $6 \mathrm{~mL} \mathrm{pc} \mathrm{kg}{ }^{-1}$ & $0,25 \mathrm{~L} \mathrm{pc} \mathrm{ha}^{-1}$ & - & $0,25 \mathrm{~L} \mathrm{pc} \mathrm{ha}^{-1}$ \\
\hline 14. $\mathrm{TS}+\mathrm{R}_{1}+\mathrm{R}_{5}$ & $6 \mathrm{~mL} \mathrm{pc} \mathrm{kg}^{-1}$ & - & $0,25 \mathrm{~L} \mathrm{pc} \mathrm{ha}^{-1}$ & $0,25 \mathrm{~L} \mathrm{pc} \mathrm{ha}^{-1}$ \\
\hline 15. $\mathrm{TS}+\mathrm{V}_{5}+\mathrm{R}_{1}+\mathrm{R}_{5}$ & $6 \mathrm{~mL} \mathrm{pc} \mathrm{kg}^{-1}$ & $0,25 \mathrm{~L} \mathrm{pc} \mathrm{ha}^{-1}$ & $0,25 \mathrm{~L} \mathrm{pc} \mathrm{ha}^{-1}$ & $0,25 \mathrm{~L} \mathrm{pc} \mathrm{ha}^{-1}$ \\
\hline
\end{tabular}

TS=tratamento de sementes.

A escala de FeHr e CAviness (1977) foi usada para identificar os estádios fenológicos da cultura antes de cada aplicação.

A altura de plantas, inserção da primeira vagem, o número de ramos e de vagens por planta (verdes, secas e total), o número de grãos por vagem, e a produtividade de grãos foram avaliadas pelos procedimentos descritos na sequência.

Altura de plantas: foi avaliada por ocasião da colheita em 10 plantas, em $1 \mathrm{~m}$ da segunda linha de cada parcela, utilizando-se fita métrica graduada em $\mathrm{cm}$.

Altura de inserção da primeira vagem: foram utilizadas as mesmas 10 plantas colhidas. Foi medida a distância da base de cada planta até da primeira vagem do caule, utilizando-se fita métrica em $\mathrm{cm}$.

Número de ramos por planta: foram contados os ramos no caule principal de cada uma das dez plantas de cada parcela.

Número de vagens por planta: determinado através de contagem das vagens verdes e secas de cada planta.

Número de grãos por vagem: as vagens foram debulhadas, sendo contados os grãos em contador eletrônico e, pela relação entre número de grãos e de vagens, foi determinado o número de grãos por vagem.

Produtividade de grãos: foi obtida na colheita das plantas de três linhas centrais em cada parcela. As plantas foram trilhadas em trilhadeira mecânica estacionária, sendo os grãos resultantes limpos e pesados. Os dados foram transformados em $k g \mathrm{ha}^{-1}$.

A análise de variância foi feita para verificar se houve pelo menos uma diferença entre as cultivares e os tratamentos e realizado contraste ortogonal para analisar as diferenças entre tratamentos. Para as variáveis número de vagens verdes, número de vagens secas, número total de vagens e número de grãos por vagem, os dados foram transformados em raiz quadrada de $x+0,5$ (BANZATTO e KRONKA, 2006).

$\mathrm{Na}$ análise estatística dos tratamentos foram formulados 14 contrastes ortogonais: $\mathrm{C} 1=+14 \mathrm{~T} 1-1 \mathrm{~T} 2-1 \mathrm{~T} 3-$ 1T4-1T5-1T6-1T7-1T8-1T9-1T10-1T11-1T12-1T13-1T14-1T15, C2=+1T1-1T2, C3=+3T1-1T3-1T4-1T5, C4=+3T2-1T3-1T41T5, C5=+2T3-1T4-1T5, C6=+1T4-1T5, C7=+2T6-1T7-1T8, $\mathrm{C} 8=+1 \mathrm{~T} 7-1 \mathrm{~T} 8, \mathrm{C} 9=+2 \mathrm{~T} 11-1 \mathrm{~T} 9-1 \mathrm{~T} 10, \mathrm{C} 10=+1 \mathrm{~T} 9-1 \mathrm{~T} 10$, $\mathrm{C} 11=+2 \mathrm{~T} 14-1 \mathrm{~T} 12-1 \mathrm{~T} 13, \quad \mathrm{C} 12=+1 \mathrm{~T} 12-1 \mathrm{~T} 13$, $\mathrm{C} 13=+1 \mathrm{~T} 3+1 \mathrm{~T} 4+1 \mathrm{~T} 5-1 \mathrm{~T} 6-1 \mathrm{~T} 7-1 \mathrm{~T} 8, \mathrm{C} 14=+1 \mathrm{~T} 3+1 \mathrm{~T} 4+1 \mathrm{~T} 5-$ 1T9-1T10-1T11. O programa estatístico utilizado foi o Sanest (ZONTA e MACHADO, 1991). 


\section{RESULTADOS E DISCUSSÃO}

Nas tabelas 2 e 3 estão os quadrados médios, valores de F, coeficientes de variação e valores médios para as variáveis em relação às cultivares. Foram observadas diferenças significativas para altura de plantas, altura de inserção da primeira vagem, vagens verdes, vagens secas, número total de vagens e produtividade de grãos; para todas estas variáveis, a cultivar Conquista proporcionou maiores resultados de que a Valiosa RR.

Don Huber e Gordon citados por Yamada (2007) observaram menor produtividade da soja resistente ao glifosato, quando tratada com glifosato, do que na isolinha convencional que não recebeu esse defensivo. Neste trabalho, não houve aplicação de glifosato em nenhuma das cultivares, no entanto foi observado menor produtividade na cultivar geneticamente modificada. Este fato pode ser atribuído a uma diferença de potencial genético produtivo entre as cultivares.

Nas tabelas 4 e 5 são apresentados os quadrados médios, valores de F, coeficientes de variação e médias de tratamentos para as variáveis. Os resultados para número de vagens secas, número total de vagens, produtividade de grãos, altura de plantas e altura de inserção da primeira vagem revelam alguma diferença significativa em relação aos tratamentos; para as duas últimas características, não foi verificada diferença estatística por contraste ortogonal entre a testemunha e os tratamentos que constaram de aplicação do bioestimulante (Tabela 6). CAmpos et al. (2007) também não observaram diferenças entre o tratamento testemunha e o tratamento com o mesmo bioestimulante utilizado neste trabalho na dose de $20 \mathrm{ml} \mathrm{L}^{-1}$ aplicado via foliar, para altura de plantas de soja. Os resultados médios para altura de plantas, ramos por planta e altura de inserção da primeira vagem foram de 83,14 e 14,4 cm respectivamente. Estes valores favorecem a colheita mecânica das plantas pois, conforme BONETTI (1983), cultivares com altura de planta igual ou superior a $65 \mathrm{~cm}$ e ponto de inserção das primeiras vagens igual ou superior a $10 \mathrm{~cm}$ são desejáveis para a realização da colheita mecânica; como se observa, os dados obtidos no trabalho foram superiores aos valores mínimos indicados pela literatura.

Tabela 2. Quadrados médios, valores de F, coeficientes de variação e valores médios para as variáveis vagens verdes, vagens secas, número total de vagens, número de grãos por vagem e produtividade de grãos, em relação às cultivares Conquista e Valiosa RR. Selvíria (MS), 2006/2007

\begin{tabular}{lccccc}
\hline Cultivares & Vagens verdes & Vagens secas & Total de vagens & Grãos por vagem & Produtividade de grãos \\
\cline { 2 - 5 } & \multicolumn{3}{c}{ unidade } & \multicolumn{2}{c}{$\mathrm{kg} \mathrm{ha}^{-1}$} \\
Conquista & 6,89 & 109,18 & 120,61 & 2,96 & 4.905 \\
Valiosa RR & 6,29 & 76,48 & 86,86 & 3,02 & 3.143 \\
\hline Q.M.Cult. & $10,76^{*}$ & $330,85^{* *}$ & $347,29^{* *}$ & 0,09 & $23.443,18^{* *}$ \\
F & 3,94 & 53,80 & 66,73 & 0,87 & 44,30 \\
CV $(\%)$ & 25,06 & 12,19 & 11,87 & 10,61 & 18,24 \\
\hline
\end{tabular}

*,**significativo a $5 \%$ e $1 \%$ de probabilidade pelo teste $\mathrm{F}$ respectivamente.

Tabela 3. Quadrados médios, valores de $\mathrm{F}$, coeficientes de variação e valores médios para as variáveis altura de plantas, ramos por planta e altura de inserção da primeira vagem nas cultivares Conquista e Valiosa RR em Selvíria (MS), 2006/2007

\begin{tabular}{lccc}
\hline Cultivares & Altura de plantas & Ramos por planta & Altura de inserção da primeira vagem \\
\hline Conquista & $\mathrm{cm}$ & unidade & $\mathrm{cm}$ \\
Valiosa RR & 86,78 & 14,10 & 13,71 \\
\hline Q.M.Cultivares & 78,60 & 13,93 & 15,08 \\
F & $2.005,73^{* *}$ & 0,8003 & $56,4440^{*}$ \\
CV $(\%)$ & 27,56 & 0,19 & 8,51 \\
\hline
\end{tabular}

*,**significativo a $5 \%$ e $1 \%$ de probabilidade pelo teste $\mathrm{F}$ respectivamente. 
Tabela 4. Quadrados médios para tratamentos, valores de F, coeficientes de variação e médias de tratamentos para as variáveis vagens verdes, vagens secas, número total de vagens, grãos por vagem e produtividade de grãos em Selvíria (MS), 2006/2007

\begin{tabular}{|c|c|c|c|c|c|}
\hline Tratamentos & Vagens verdes & Vagens secas & Total de vagens & Grãos por vagem & Produtividade de grãos \\
\hline & & $-\mathrm{ur}$ & dade - & & $\mathrm{kg} \mathrm{ha}^{-1}$ \\
\hline 01. Testemunha & 8,8 & 72,4 & 81,5 & 2,1 & 2.578 \\
\hline 02. TS & 8,9 & 97,2 & 106,3 & 1,9 & 4.331 \\
\hline 03. $\mathrm{V}_{5}$ & 9,2 & 80,1 & 89,4 & 2,7 & 4.382 \\
\hline 04. $\mathrm{R}_{1}$ & 11,9 & 114,9 & 127,0 & 1,8 & 4.500 \\
\hline 05. $R_{5}$ & 11,6 & 98,2 & 110,2 & 1,6 & 3.540 \\
\hline 06. $\mathrm{TS}+\mathrm{V}_{5}$ & 12,1 & 89,5 & 101,7 & 2,0 & 3.984 \\
\hline 07. $\mathrm{TS}+\mathrm{R}_{1}$ & 11,4 & 114,5 & 122,5 & 1,9 & 4.391 \\
\hline 08. $\mathrm{TS}+\mathrm{R}_{5}$ & 10,3 & 97,6 & 108,1 & 1,9 & 4.987 \\
\hline 09. $V_{5}+R_{1}$ & 13,6 & 77,5 & 94,8 & 1,9 & 3.510 \\
\hline 10. $V_{5}+R_{5}$ & 7,4 & 79,7 & 87,3 & 1,9 & 3.395 \\
\hline 11. $R_{1}+R_{5}$ & 10,0 & 83,2 & 93,4 & 2,4 & 4.479 \\
\hline 12. $\mathrm{TS}+\mathrm{V}_{5}+\mathrm{R}_{1}$ & 13,2 & 90,8 & 104,7 & 2,1 & 3.201 \\
\hline 13. $\mathrm{TS}+\mathrm{V}_{5}+\mathrm{R}_{5}$ & 9,7 & 98,9 & 108,9 & 2,1 & 4.428 \\
\hline 14. $\mathrm{TS}+\mathrm{R}_{1}+\mathrm{R}_{5}$ & 9,0 & 93,4 & 102,6 & 1,8 & 4.345 \\
\hline 15. $\mathrm{TS}+\mathrm{V}_{5}+\mathrm{R}_{1}+\mathrm{R}_{5}$ & 13,1 & 99,3 & 112,6 & 1,7 & 3.957 \\
\hline Q.M.Trat. & 2,5958 & 0,2568 & 12,3213 & 0,2568 & 866,781 \\
\hline $\mathrm{F}$ & 0,95 & $2,54^{*}$ & $53,80^{* *}$ & 2,54 & $1,63^{*}$ \\
\hline $\mathrm{CV}(\%)$ & 25,0 & 10,61 & 12,2 & 10,6 & 18,24 \\
\hline
\end{tabular}

*,**significativo a $5 \%$ e $1 \%$ de probabilidade pelo teste $\mathrm{F}$ respectivamente.

Tabela 5. Quadrados médios para tratamentos, valores de F, coeficientes de variação e médias de tratamentos para as variáveis altura de plantas, número de ramos por planta e altura de inserção da primeira vagem em Selvíria (MS), $2006 / 2007$

\begin{tabular}{|c|c|c|c|}
\hline Tratamentos & Altura de plantas & Ramos por planta & Altura de inserção da primeira vagem \\
\hline & $\mathrm{cm}$ & unidade & $\mathrm{cm}$ \\
\hline 01. Testemunha & 85,36 & 14,45 & 15,27 \\
\hline 02. TS & 75,56 & 12,99 & 12,80 \\
\hline 03. $\mathrm{V}_{5}$ & 80,79 & 13,86 & 14,56 \\
\hline 04. $\mathrm{R}_{1}$ & 87,77 & 14,24 & 15,65 \\
\hline 05. $\mathrm{R}_{5}$ & 86,42 & 14,86 & 13,66 \\
\hline 06. $\mathrm{TS}+\mathrm{V}_{5}$ & 65,71 & 13,27 & 11,87 \\
\hline 07. $\mathrm{TS}+\mathrm{R}_{1}$ & 78,70 & 14,91 & 14,24 \\
\hline 08. $\mathrm{TS}+\mathrm{R}_{5}$ & 90,10 & 14,11 & 13,54 \\
\hline 09. $V_{5}+R_{1}$ & 89,47 & 13,66 & 15,79 \\
\hline 10. $V_{5}+R_{5}$ & 91,95 & 14,00 & 16,17 \\
\hline 11. $R_{1}+R_{5}$ & 89,20 & 16,41 & 15,65 \\
\hline 12. $\mathrm{TS}+\mathrm{V}_{5}+\mathrm{R}_{1}$ & 79,22 & 13,07 & 16,06 \\
\hline 13. $\mathrm{TS}+\mathrm{V}_{5}+\mathrm{R}_{5}$ & 70,14 & 13,40 & 15,07 \\
\hline 14. $\mathrm{TS}+\mathrm{R}_{1}+\mathrm{R}_{5}$ & 79,17 & 13,87 & 12,20 \\
\hline 15. $T S+V_{5}+R_{1}+R_{5}$ & 90,74 & 13,15 & 13,44 \\
\hline Q.M.Trat. & 503,3893 & 6,4574 & 16,1306 \\
\hline $\mathrm{F}$ & $6,9164^{* *}$ & 1,5436 & $2,4319^{* *}$ \\
\hline $\mathrm{CV}(\%)$ & 10,3 & 14,6 & 17,9 \\
\hline
\end{tabular}

*,**significativo a $5 \%$ e $1 \%$ de probabilidade pelo teste $\mathrm{F}$ respectivamente. 
O número de vagens verdes na ocasião da colheita não foi alterado pela aplicação do produto indicando que este não influenciou a maturação das vagens. Também não houve alteração significativa no número de grãos por vagem pela aplicação, e o número médio foi de duas sementes por vagem.

Na tabela 6, observam-se os quadrados médios e teste $\mathrm{F}$ para contrastes entre tratamentos para altura de plantas, altura de inserção da primeira vagem, número total de vagens e produtividade de grãos. Foram observadas diferenças entre a parcelatestemunha e as parcelas que receberam algum tratamento com o produto para as variáveis número de vagens secas, número total de vagens e produtividade de grãos. Os desdobramentos destes contrastes estão apresentados na tabela 7. Não foi observada diferença estatística entre aplicação via sementes e via foliar do produto nas variáveis estudadas. Em plantas de milho, a aplicação do fitorreguladores foi mais eficiente quando executada no tratamento de sementes em comparação com a pulverização foliar (Dourado Neto et al., 2004).

Para produção de vagens secas, a aplicação de bioestimulante proporcionou incremento de $23 \%$ em relação à testemunha, a aplicação via sementes,
$25 \%$ e a aplicação via foliar, $26 \%$. Valores proporcionais foram obtidos para o número total de vagens, e houve diferença entre a aplicação do produto no estádio vegetativo $V_{5}$ e nos estádios reprodutivos, $R_{1}$ e $R_{5}$. $O$ número de vagens por planta foi superior nas aplicações nos estádios reprodutivos em relação ao estádio vegetativo em $25 \%$ e $24 \%$, respectivamente, para número de vagens secas e número total de vagens. Совиссі et al. (2005), estudando respostas do feijoeiro à aplicação de bioestimulante ressaltaram a importância da fase fenológica da planta no momento da aplicação, visto que o bioestimulante aplicado na mesma dose em estádios fenológicos diferentes não proporcionou os mesmos resultados para produtividade; no feijoeiro, observaram-se maiores resultados para aplicação em $R_{5}$ em relação a $V_{4}$.

Os resultados da aplicação do produto no estádio $R_{1}$ e no $R_{5}$ não diferiram entre si. Em relação ao número de aplicações foliares houve diferença entre uma aplicação do produto e duas aplicações do produto (contraste ortogonal 14), com incremento da realização de uma única aplicação em relação a duas aplicações de $3 \%$ e $2 \%$ para vagens secas e número total de vagens.

Tabela 6. Quadrados médios para contraste $\mathrm{F}$ entre tratamentos para as variáveis altura de plantas, altura de inserção da primeira vagem, número de vagens secas, número total de vagens, número de grãos por vagem e produtividade de grãos. Selvíria (MS), 2006/07

\begin{tabular}{|c|c|c|c|c|c|}
\hline Contrastes $\left({ }^{1}\right)$ & $\begin{array}{c}\text { Altura } \\
\text { de plantas }\end{array}$ & $\begin{array}{l}\text { Altura de inserção } \\
\text { da primeira vagem }\end{array}$ & Vagens secas & Total de vagens & $\begin{array}{c}\text { Produtividade } \\
\text { de grãos }\end{array}$ \\
\hline & \multicolumn{2}{|c|}{$\mathrm{cm}$} & \multicolumn{2}{|c|}{ unidade } & $\mathrm{kg} \mathrm{ha}^{-1}$ \\
\hline $\mathrm{C} 1$ & 61,2193 & 6,5750 & $40,4685^{* *}$ & $43,1526^{* *}$ & $5.162,8200^{* *}$ \\
\hline $\mathrm{C} 2$ & 13,5998 & $41,0900^{* *}$ & $29,1289 *$ & $26,2395^{*}$ & $3.615,9026^{*}$ \\
\hline $\mathrm{C} 3$ & $584,7358 * *$ & 6,9646 & $43,7672^{* *}$ & $45,6131^{* *}$ & $4361,9295^{* *}$ \\
\hline $\mathrm{C} 4$ & $2.609,3777^{* *}$ & $48,1667 * *$ & 0,0001 & 0,2304 & 57,7890 \\
\hline C5 & $326,0418^{*}$ & 18,6252 & $39,6042^{*}$ & $43,3093^{* *}$ & 180,6484 \\
\hline C6 & 47,6008 & 18,1302 & 10,4166 & 9,5200 & 921,2758 \\
\hline $\mathrm{C} 7$ & 212,5209 & 0,0469 & 14,6548 & 8,9532 & 603,2606 \\
\hline $\mathrm{C} 8$ & 7,2900 & 15,8006 & 10,8573 & 7,1255 & 303,0365 \\
\hline C9 & 6,4533 & 0,0833 & 1,3940 & 0,3185 & $1.422,4133$ \\
\hline C10 & 30,2499 & 1,1025 & 0,2640 & 2,4220 & 15,2336 \\
\hline C11 & $1.862,5209^{* *}$ & 21,6008 & 0,1127 & 0,9130 & 406,7409 \\
\hline $\mathrm{C} 12$ & $519,8402 * *$ & 1,9599 & 2,8185 & 0,6800 & $1.585,1690$ \\
\hline C13 & 111,3252 & $31,3633^{*}$ & 1,1163 & 0,5534 & 278,2850 \\
\hline $\mathrm{C} 14$ & 326,7054 & 33,0625 & $39,6314^{* *}$ & $32,7061^{*}$ & 370,7850 \\
\hline Resíduo & 72,7825 & 6,6328 & 5,2041 & 6,1488 & 529,1346 \\
\hline
\end{tabular}

*,**significativo a $5 \%$ e $1 \%$ de probabilidade pelo teste $\mathrm{F}$ respectivamente.

( $\left.{ }^{1}\right) \mathrm{C} 1=+14 \mathrm{~T} 1-1 \mathrm{~T} 2-1 \mathrm{~T} 3-1 \mathrm{~T} 4-1 \mathrm{~T} 5-1 \mathrm{~T} 6-1 \mathrm{~T} 7-1 \mathrm{~T} 8-1 \mathrm{~T} 9-1 \mathrm{~T} 10-1 \mathrm{~T} 11-1 \mathrm{~T} 12-1 \mathrm{~T} 13-1 \mathrm{~T} 14-1 \mathrm{~T} 15, \mathrm{C} 2=+1 \mathrm{~T} 1-1 \mathrm{~T} 2, \mathrm{C} 3=+3 \mathrm{~T} 1-1 \mathrm{~T} 3-1 \mathrm{~T} 4-1 \mathrm{~T} 5, \mathrm{C} 4=+3 \mathrm{~T} 2-1 \mathrm{~T} 3-1 \mathrm{~T} 4-1 \mathrm{~T} 5$, $\mathrm{C} 5=+2 \mathrm{~T} 3-1 \mathrm{~T} 4-1 \mathrm{~T} 5, \mathrm{C} 6=+1 \mathrm{~T} 4-1 \mathrm{~T} 5, \mathrm{C} 7=+2 \mathrm{~T} 6-1 \mathrm{~T} 7-1 \mathrm{~T} 8, \mathrm{C} 8=+1 \mathrm{~T} 7-1 \mathrm{~T} 8, \mathrm{C} 9=+2 \mathrm{~T} 11-1 \mathrm{~T} 9-1 \mathrm{~T} 10, \mathrm{C} 10=+1 \mathrm{~T} 9-1 \mathrm{~T} 10, \mathrm{C} 11=+2 \mathrm{~T} 14-1 \mathrm{~T} 12-1 \mathrm{~T} 13, \mathrm{C} 12=+1 \mathrm{~T} 12-$ $1 \mathrm{~T} 13, \mathrm{C} 13=+1 \mathrm{~T} 3+1 \mathrm{~T} 4+1 \mathrm{~T} 5-1 \mathrm{~T} 6-1 \mathrm{~T} 7-1 \mathrm{~T} 8, \mathrm{C} 14=+1 \mathrm{~T} 3+1 \mathrm{~T} 4+1 \mathrm{~T} 5-1 \mathrm{~T} 9-1 \mathrm{~T} 10-1 \mathrm{~T} 11$ 
Tabela 7. Número relativo de vagens secas por planta, total de vagens por planta e produtividade de grãos em função dos contrastes ortogonais significativos entre os tratamentos

\begin{tabular}{|c|c|}
\hline Vagens secas por planta & Incremento \\
\hline $\mathrm{C} 1=$ Testemunha $(\mathrm{T})$ vs. Bioestimulante $(\mathrm{B})$ & $23 \%$ em relação à Testemunha \\
\hline $\mathrm{C} 2=\mathrm{T}$ vs. $\mathrm{B}$ via sementes & $25 \%$ em relação à Testemunha \\
\hline $\mathrm{C} 3=\mathrm{T}$ vs. $\mathrm{B}$ via foliar & $26 \%$ em relação à Testemunha \\
\hline$C 5=B$ em $V_{5}$ vs. $B$ em $R_{1}$ e $R_{5}$ & $25 \%$ em relação à aplic. em $V_{5}$ \\
\hline C14=1 aplicação foliar de B vs. 2 aplic. foliares de B & $3 \%$ em relação à 2 aplic. foliares \\
\hline Total de vagens por planta & Incremento \\
\hline $\mathrm{C} 1=$ Testemunha $(\mathrm{T})$ vs. Bioestimulante $(\mathrm{B})$ & $22 \%$ em relação à Testemunha \\
\hline $\mathrm{C} 2=\mathrm{T}$ vs. $\mathrm{B}$ via sementes & $30 \%$ em relação à Testemunha \\
\hline $\mathrm{C} 3=\mathrm{T}$ vs. $\mathrm{B}$ via foliar & $25 \%$ em relação à Testemunha \\
\hline$C 5=B$ em $V_{5}$ vs. B em $R_{1}$ e $R_{5}$ & $24 \%$ em relação à aplic. em $V_{5}$ \\
\hline C14= 1 aplicação foliar de B vs. 2 aplic. foliares de B & $2 \%$ em relação à 2 aplic. foliares \\
\hline Produtividade relativa de grãos & Incremento \\
\hline $\mathrm{C} 1=$ Testemunha $(\mathrm{T})$ vs. Bioestimulante $(\mathrm{B})$ & $37 \%$ em relação à Testemunha \\
\hline $\mathrm{C} 2=\mathrm{T}$ vs. $\mathrm{B}$ via sementes & $40 \%$ em relação à Testemunha \\
\hline $\mathrm{C} 3=\mathrm{T}$ vs. $\mathrm{B}$ via foliar & $37 \%$ em relação à Testemunha \\
\hline
\end{tabular}

Fresoli et al. (2006) estudando efeito de bioestimulante em soja também não observaram diferença entre o tratamento de sementes e aplicação foliar no estádio $V_{5}$ para produtividade, obtendo maiores produtividades com a aplicação do produto tanto em aplicação foliar quanto via sementes em relação à testemunha, obtendo produtividade aproximada de $3.205 \mathrm{~kg} \mathrm{ha}^{-1}$ para esses tratamentos.

A produtividade de grãos foi incrementada em $37 \%$ com a utilização do bioestimulante em relação à testemunha, $40 \%$ em relação à aplicação do produto via sementes e $37 \%$ em relação à aplicação via foliar. Esses resultados corroboram os de VIEIRA et al. (2005) que consideraram que o bioestimulante na concentração de $10 \mathrm{~mL} \mathrm{~kg}^{-1}$ de sementes incrementa em $24,3 \%$ a produtividade de grãos de soja. Também Milléo (2000) obteve maiores produções de vagens e de grãos por planta de soja em aplicação via sementes e foliar no estágio $V_{5}$, e esta última aplicação proporcionou incremento de $65 \%$ em relação à testemunha. BRACCINI et al. (2005), trabalhando com soja e bioestimulante, que possui os mesmos ingredientes do produto utilizado neste trabalho, porém em concentração 10 vezes maior, obtiveram a maior produtividade com o tratamento com a maior dose do produto aplicado via foliar $\left(75 \mathrm{~mL} \mathrm{ha}^{-1}\right)$, com incremento superior a $92 \%$ em relação à testemunha.

A realização de uma única aplicação do produto durante o ciclo da cultura incrementa a produtividade seja com aplicação via sementes seja foliar em quaisquer dos estádios fenológicos estudados. Para a produção de vagens, foi observada diferença estatística entre os estádios vegetativo e reprodutivo, tornando interessante o estudo adicional do estádio fenológico para aplicação do produto sobre a qualidade dos grãos obtidos mesmo que isso não tenha sido observado para produtividade de grãos.

\section{CONCLUSÃO}

O bioestimulante proporciona incremento no número de vagens por planta e produtividade de grãos tanto em aplicação via sementes quanto via foliar, confirmando a hipótese deste estudo. Todavia, a maior produtividade não está relacionada ao maior crescimento da parte aérea, considerando-se a altura das plantas, ramos por planta, altura de inserção da primeira vagem. Em relação ao aumento da produtividade, o bioestimulante é mais efetivo quando aplicado na fase reprodutiva.

\section{AGRADECIMENTOS}

Agradecemos à Coordenação de Aperfeiçoamento de Pessoal de Nível Superior, CAPES, pelo fornecimento de bolsa de mestrado, e à Fundação de Apoio à Pesquisa Agropecuária de Chapadão localizada na cidade de Chapadão do Sul (MS), pelo fornecimento das sementes das cultivares de soja utilizadas neste experimento. 


\section{REFERÊNCIAS}

BANZATTO, D.A.; KRONKA, S.N. Experimentação agrícola. 4. ${ }^{\mathrm{a}}$ ed. Jaboticabal: FUNEP, 2006. 237 p.

BONETTI, L.P. Cultivares e seu melhoramento genético. In: VERNETTI, F. J. (coord.). Soja: genética e melhoramento. Campinas: Fundação Cargill, 1983. p. 741-794.

BRACCINI, A.L.; MONFERDINI, M.A.; ÁVILA, M.R.; SCAPIM, C.A.; BRAMBILLA, D.; ARAGÃO, R.M.; BRAMBILLA, T. Emergência das plântulas e componentes da produção de sementes em resposta a diferentes doses e formas de aplicação do bioestimulante Stimulate 10X na cultura da soja. In.: REUNIÃO DE PESQUISA DE SOJA DA REGIÃO CENTRAL DO BRASIL, 27., 2005, Cornélio Procópio. Resumos ... Londrina: Embrapa Soja, 2005. p.565-566.

CAMPOS, M.F.; ONO, E.O.; LIMA, G.P.P.; RODRIGUES, J.D. Desenvolvimento de plantas de soja em resposta aos reguladores vegetais. Revista Brasileira de Biociências, v.5, p.9-11, 2007.

CARVALHO, L.H.; CHIAVEGATO, E.J.; CIA, E.; KONDO, J.I.; SABINO, J.C.; PETTINELLI JÚNIOR, A.; BORTOLETTO, N.; GALLO, P.B. Fitorreguladores de crescimento e capação na cultura algodoeira. Bragantia, v.53, 1994.

CASTRO, P.R.C. Agroquímicos de controle hormonal na agricultura tropical. Piracicaba, 2006. 46p. (Série Produtor Rural n.32)

CASTRO, P.R.C.; VELLO, N.A. Ação de fitorreguladores no desenvolvimento da soja cultivar Davis. Anais da Escola Superior de Agricultura "Luiz de Queiroz", v.38, p.269-279, 1981.

COBUCCI, T.; RUCK, F.J. W.; SILVA, J.G. Resposta do feijoeiro (Phaseolus vulgaris L.) às aplicações de bioestimulante e complexos nutritivos. In: CONAFE, Congresso Nacional de Pesquisa de Feijão, 8., 2005, Goiânia, Anais... Santo Antonio de Goiás: Embrapa Arroz e Feijão, 2005. p.1078-1081.

CROZIER, A.; KAMIYA, Y.; BISHOP, G.; YOKOTA, T. Biosynthesis of hormones and elicitor molecules. In: BUCHANAN, B.B.; GRISSEN, W.; JONES, R.L. (Ed.) Biochemistry and Molecular Biology of Plants. Maryland: Amercian Society of Plant Physiologists, 2000. p. 850-894.

DOURADO NETO, D.; DARIO, G.J.A.; VIEIRA JÚNIOR, P.A.; MANFRON, P.A.; MARTIN, T.N.; BONNECARRÉRE, R.A.G.; CRESPO, P.E.N. Aplicação e influência do fitorregulador no crescimento das plantas de milho. Revista da FZVA, v.11, p.19, 2004.

EMBRAPA. Empresa Brasileira de Pesquisa Agropecuária. Sistema Brasileiro de Classificação de Solos. Rio de Janeiro: EMBRAPA/CNPSO, 1999. 412 p.

EMBRAPA. Empresa Brasileira de Pesquisa Agropecuária. Centro Nacional de Pesquisa de Soja. Recomendações técnicas para a cultura da soja na Região Central do Brasil 1996/97. Londrina: Embrapa, CNPSo, 1996. 149 p. (Documentos 88)
FEHR, W. R.; CAVINESS, C. E. Stages of soybean development. Ames: Cooperative Extension Service-Iowa, State University, 1977. 11p. (Special Report, 80)

FRESOLI, D.M.; BERET, P.N.; GUAITA, S.J.; ROJAS, P.H. Evalución de un bioestimulante en sojas con distintos hábitos de crecimento. In: MERCOSOJA 2006. CONGRESO DE SOJA DEL MERCOSUR, 3., 2006, Rosario. Anais... Rosario: Mercosoja 2006, 2006. p. 578-581.

HARPER, D. In the field with herbicide resistant crops: Roundup Ready soybeans. Proceedings of the Western Society of Weeed Science, p.8.1997.

LARCHER, W. Ecofisiologia vegetal. São Carlos: Rima, 2006. p.295-338.

MASCARENHAS, H.A.A.; TANAKA, R.T. Soja. In: RAIJ, B.van; CANTARELLA, H.; QUAGGIO, J.A.; FURLANI, A.M.C. (Ed.). Recomendações de adubação e calagem para o Estado de São Paulo. 2.ed. Campinas: IAC, FUNDAG, 1997. p.202-203.

MILLÉO, M. V. R. Avaliação da eficiência agronômica do produto Stimulate aplicado no tratamento e em pulverização foliar sobre a cultura da soja (Glycine Max L.). Ponta Grossa: Universidade Estadual de Ponta Grossa, 2000. 18p. (Relatório técnico)

SANTOS, C.M.G.; VIEIRA, E.L. Efeito de bioestimulante na germinação de grãos, vigor de plântulas e crescimento inicial do algodoeiro. Magistra, v.17, p.124-130, 2005.

TAIZ, L.; ZEIGER, E. Fisiologia Vegetal. 3. a ed., Porto Alegre: Artmed, 2004. 559p.

VIEIRA, E. L.; CASTRO, P. R. C.; CATO, S. C.; SILVA, g P. Stimulate no sistema de produção da soja. In: REUNIÃO DE PESQUISA DE SOJA NA REGIÃO CENTRAL DO BRASIL, 27. 2005, Cornélio Procópio. Resumos... Cornélio Procópio, 2005. p.82-83.

YAMADA, T. Por que há tantas doenças de plantas no sistema plantio direto? Informações Agronômicas, v.117, 30 p., 2007.

ZONTA, E. P.; MACHADO, A. A. SANEST-Sistema de análise de variância para microcomputadores. Pelotas: UFPel, 1991. $104 p$. 